\title{
DES LEVIERS DUACIION POUR AIDER LA RECONNAISSANCE DE LA FORMATION GÉNÉRALE DES ADULTES
}

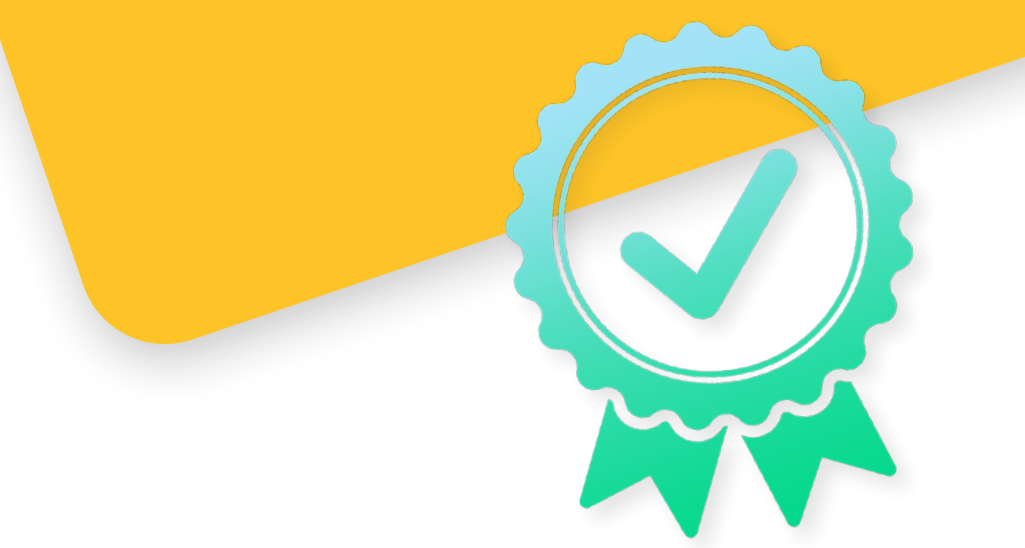
http://creativecommons.org/licences/by/4.0/ deed.fr

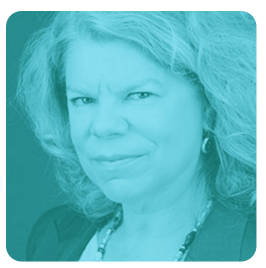

BRIGITTE VOYER

Professeure Titulaire

Université du Québec à Montréal

Chercheure du CRIFPE

La reconnaissance professionnelle des enseignants de la formation générale des adultes (FGA) est une source de préoccupation. Elle n'est pas étrangère au manque de reconnaissance sociale de l'ensemble du secteur. Quels moyens peuvent aider à son développement? Cette question est abordée ici avec l'idée qu'une profession est un construit social. Une profession s'élabore à long terme avec la contribution d'acteurs de divers horizons. Chacun d'eux dispose, à son niveau, de leviers d'action. Un levier particulièrement puissant relève du ministère de l'Éducation et des universités : permettre l'accès à des savoirs spécialisés et reconnaître l'expertise. Or, aucune université québécoise n'offre une formation spécialisée de ler cycle pour préparer spécifiquement à l'enseignement à la FGA (Voyer, Brodeur, Meilleur et al., 2012). Cependant, d'autres leviers peuvent améliorer la reconnaissance de la FGA. En voici trois qui interpellent d'autres acteurs.

\section{PRATIQUER L'ANDRAGOGIE AU QUOTIDIEN : UN LEVIER POUR LE PERSONNEL ENSEIGNANT}

La FGA s'adresse à des personnes considérées comme des adultes. Aucun apprenant n'est soumis à l'obligation de se former. Ceci constitue le premier noyau d'identification commun à l'ensemble des intervenants de ce secteur éducatif. L'acte d'enseigner est destiné à des personnes volontaires. Cette spécificité propre à la FGA devrait se traduire dans la pratique. Ensuite, l'enseignement à la FGA se réalise au sein d'une infrastructure et d'un fonctionnement interne sans équivalent à la formation générale des jeunes : l'entrée continue et les sorties variables, les classes multiniveaux et l'enseignement modulaire individualisé. Un tel contexte exige d'adopter une posture enseignante particulière. Une recherche récente (Voyer, Ouellet, Mercier et Ouellet, 2021) suggère que certaines personnes enseignantes adoptent plus facilement que d'autres une posture relevant des principes de l'andragogie. Leur " posture d'accompagnement»" est plus conforme aux orientations des programmes d'études de la FGA. Celle-ci diffère de la "posture de contrôle». Une posture d'enseignement est une manière d'affirmer les particularités d'un univers professionnel. Elle est un révélateur de l'identité professionnelle. Orienter son enseignement selon les orientations de la FGA est un levier pertinent pour distinguer sa pratique de celle exercée dans d'autres secteurs éducatifs et pour dévoiler une expertise.

\section{GÉRER UNE DEMANDE DE FORMATION DIVERSIFIÉE : UN LEVIER POUR LES GESTIONNAIRES}

Nous reconnaissons qu'il s'avère difficile d'appliquer pleinement les principes andragogiques, tellement la proportion de jeunes adultes est importante à la FGA. Un changement effectué à la Loi sur l'instruction publique en 1989 a favorisé le passage direct de la formation générale des jeunes vers la FGA. Ceci a engendré l'exode des jeunes vers la FGA. Selon Doray et Bélanger 
(2014 : 245-247), ce changement législatif a contribué à substituer la mission même de la formation générale des adultes. Occupé à la lutte au décrochage scolaire et à la diplomation de jeunes en difficulté, le secteur a délaissé sa vocation première d'éducation des adultes. Or, cette substitution de mission contribue à rendre flove l'identité même de la FGA. Ceci n'aide en rien sa reconnaissance sociale. Alors, que peuvent y faire les gestionnaires? Selon ces chercheurs, la présence massive des jeunes à la FGA comble facilement le nombre de places dans les centres d'éducation des adultes : la gestion des inscriptions en serait facilitée. Pour les gestionnaires, il serait moins nécessaire qu'autrefois de susciter la demande de formation auprès d'une diversité d'adultes, orientation pourtant préconisée par la Politique d'éducation des adultes et de formation continue (MEQ, 2002). Les gestionnaires pourraient prévoir dans leur projet éducatif des mesures pour assurer l'inscription d'adultes de tous les âges dans les divers services d'enseignement. Ceci pourrait contribuer à préserver la mission fondamentale de la FGA. Puis, en utilisant davantage leur pouvoir de représentation, ils seraient en mesure de réclamer auprès des instances ministérielles des mesures de gestion encore plus efficaces pour susciter et répondre à une demande de formation plus variée, selon un principe d'équité. Pour ce faire, le recours aux associations de gestionnaires s'avère pertinent pour communiquer avec les décideurs. Par exemple, la Table des responsables de l'éducation des adultes du Québec (TREAQ) offre une visibilité utile à la reconnaissance de la FGA.

\section{AMÉLIORER LES CONDITIONS DE TRAVAIL: UN LEVIER POUR LES SYNDICATS ET LES ASSOCIATIONS}

La qualité des conditions de travail fait souvent écho à la reconnaissance d'une profession. Or, le personnel enseignant de la FGA est le plus précaire du réseau éducatif : plus de $70 \%$ n'ont pas de statut d'emploi régulier. L'instabilité d'emploi peut réduire l'investissement dans des activités collectives. À cet égard, les syndicats d'enseignants sont des acteurs de premier plan pour améliorer leurs conditions. Or, on peut penser que les associations syndicales seront plus susceptibles d'obtenir des gains significatifs, si les enseignants de la FGA s'y investissent plus résolument. Leur efficacité peut être augmentée si le personnel y délègue des collègues soucieux de l'action collective et pleinement mobilisés. En effet, l'influence des syndicats se nourrit en bonne partie de l'investissement de ses membres. D'autres réseaux collectifs portent eux aussi la voix des préoccupations exprimées à la FGA. Pensons, à l'Association québécoise des intervenantes et intervenants de la FGA (AQIFGA) et à I'Institut de Coopération en éducation des adultes (ICEA) qui contribuent à la visibilité du réseau.

Il semble que la reconnaissance professionnelle des enseignants va de pair avec la reconnaissance sociale du secteur de la FGA. Bien sûr, les universités et les décideurs détiennent une clef essentielle à cette double reconnaissance en diffusant et en légitimant le savoir professionnel. Or, chaque catégorie d'acteurs, à son niveau, peut aussi investir les mécanismes propres à leur milieu. Ceux-ci affirment ainsi les spécificités de l'enseignement aux adultes et contribuent à l'identité collective.

\section{EN SAVOIR PLUS}

- Doray, P. et Bélanger, P. (2014). Retirer à Pierrette pour donner à Alexandre! Le développement de la formation générale des adultes au Québec.
Revue des sciences de l'éducation, 40(2), 215-251. https://doi.org/10.7202/1028420ar
- Voyer, B, Ouellet, C, Mercier, J-P et Ouellet, S. (2021). Analyse de pratiques d'enseignement en lecture et en écriture à la formation générale des
adultes (FGA) : une recherche-action menée en collaboration pour aider les apprenants à améliorer leurs compétences en français écrit.
Rapport de recherche scientifique, Fonds de recherche du Québec-Société et culture (FRQSC). Action concertée Programme lecture et
écriture. Rapport no 2018-LC-211018. https://fra.gouv.ca/app/uploads/2021/08/brigitte.voyer_rapport_ecriture-lecture.pdf
- Voyer, B., Brodeur, M., Meilleur, J.-F. et Sous-comité de la Table MELS-Universités de la formation à l'enseignement des adultes. (2012). État de la
programmes actuels de formation à l'enseignement au Québec. Analyse, constats et pistes de solution. Document de travail. Rapport final
préparé par les membres du sous-comité de la Table MELS-Universités sur la formation à l'enseignement à la formation générale des
adultes. Montréal: 25 mai 2012. Récupéré de http://bv.cdeacf.ca/EA_PDF/159015.pdf.

POUR CITER CETTE FICHE

Voyer, B. (2021). Des leviers d'action pour aider la reconnaissance de la Formation générale des adultes. Ce que nous apprend la recherche (CRIFPE), 1(6). https://doi.org/10.18162/cqnalr.2021.1.6 